Supplement of Clim. Past, 17, 1443-1454, 2021

https://doi.org/10.5194/cp-17-1443-2021-supplement

(C) Author(s) 2021. CC BY 4.0 License.

(c) (i)

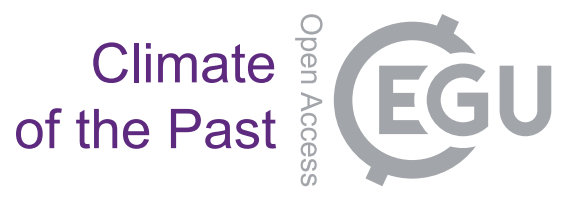

Supplement of

\title{
Precise timing of MIS 7 substages from the Austrian Alps
}

Kathleen A. Wendt et al.

Correspondence to: Kathleen A. Wendt (kathleen.wendt@oregonstate.edu)

The copyright of individual parts of the supplement might differ from the article licence. 

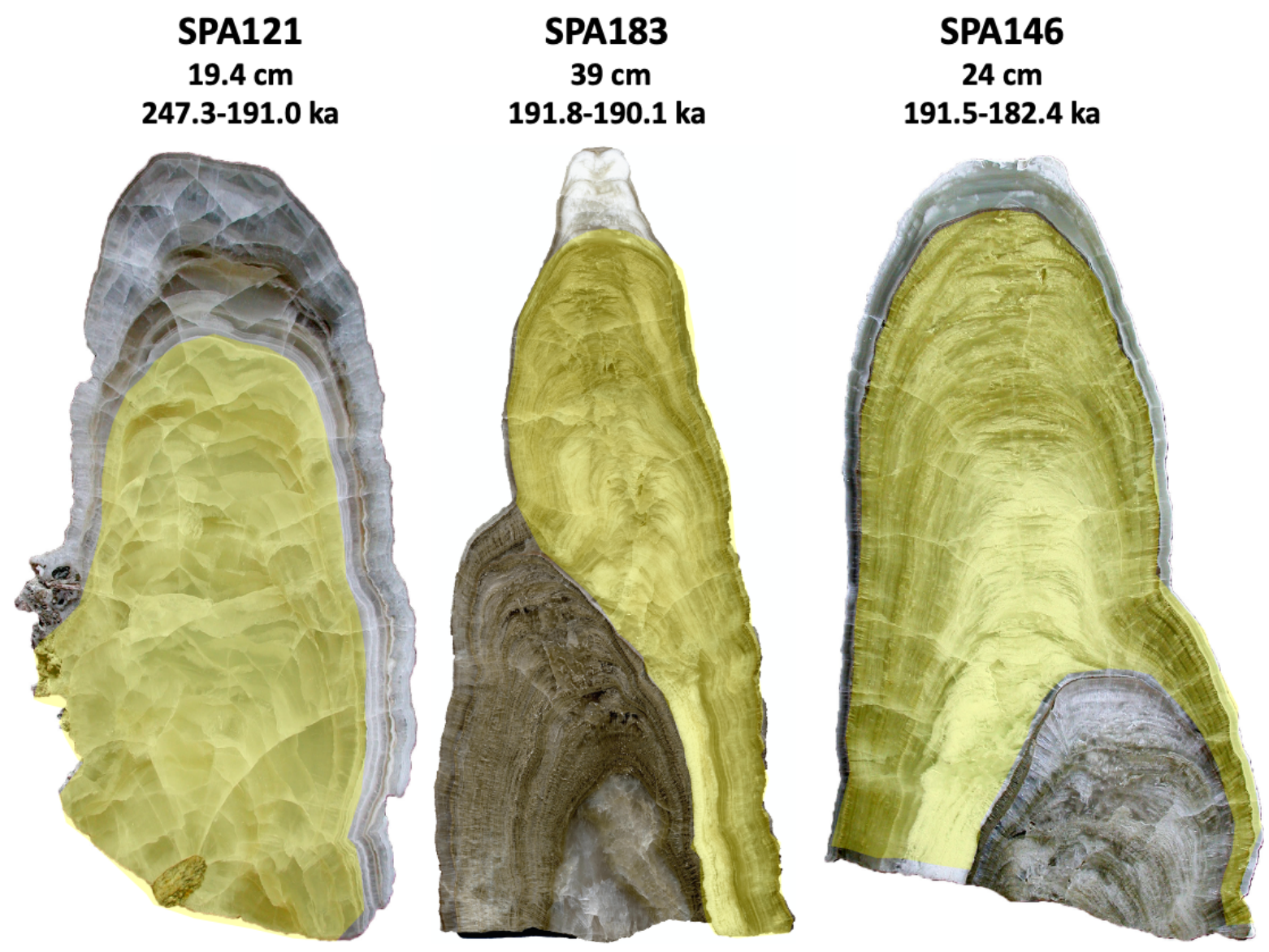

2

3 Figure S1: Stalagmites SPA121, 183, and 146. MIS 7 growth sections examined in this study are highlighted in 4 yellow. 

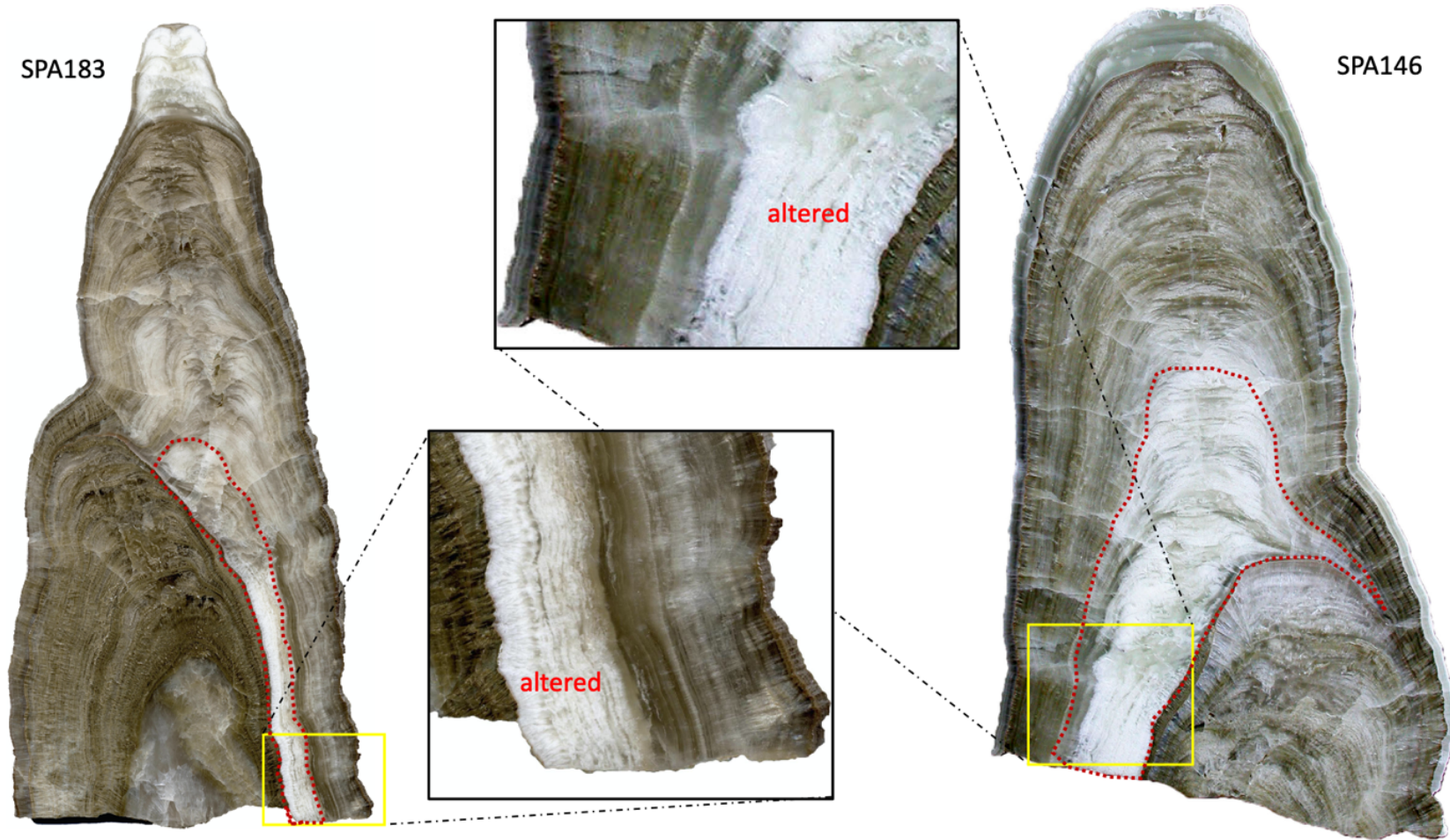

8 Figure S2: Diagenetically altered portions of SPA183 and 146 highlighted by dashed red lines.

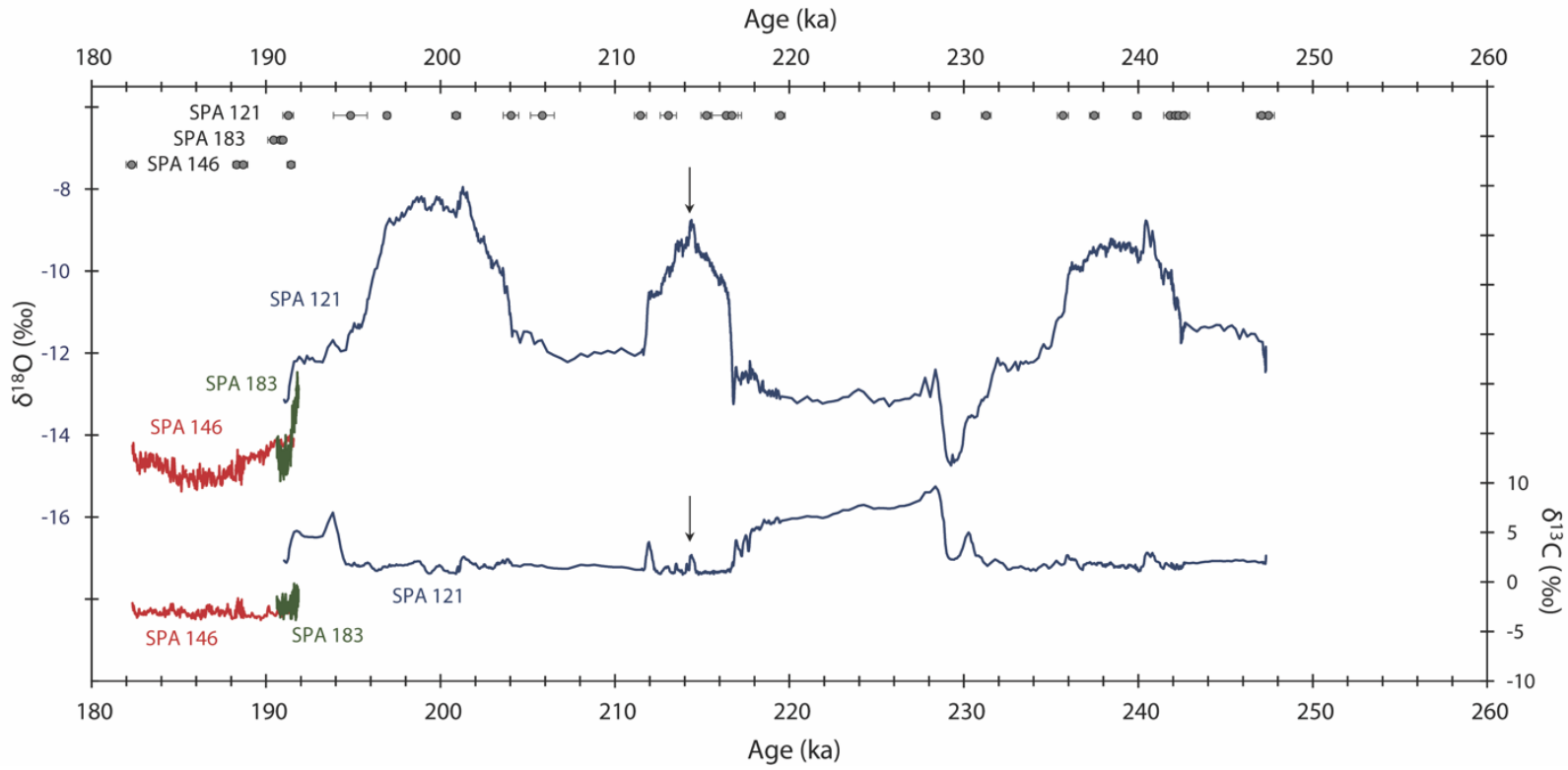

Figure S3: $\delta^{18} \mathrm{O}$ and $\delta^{13} \mathrm{C}$ records of stalagmite SPA121 (blue) compared to the shorter growth intervals of stalagmites SPA183

15 (green) and SPA146 (red). At top, U-Th age results with associated 2 sigma uncertainties. Black arrows indicate location of the 16 dust layer in stalagmite SPA121 (see text). 
17
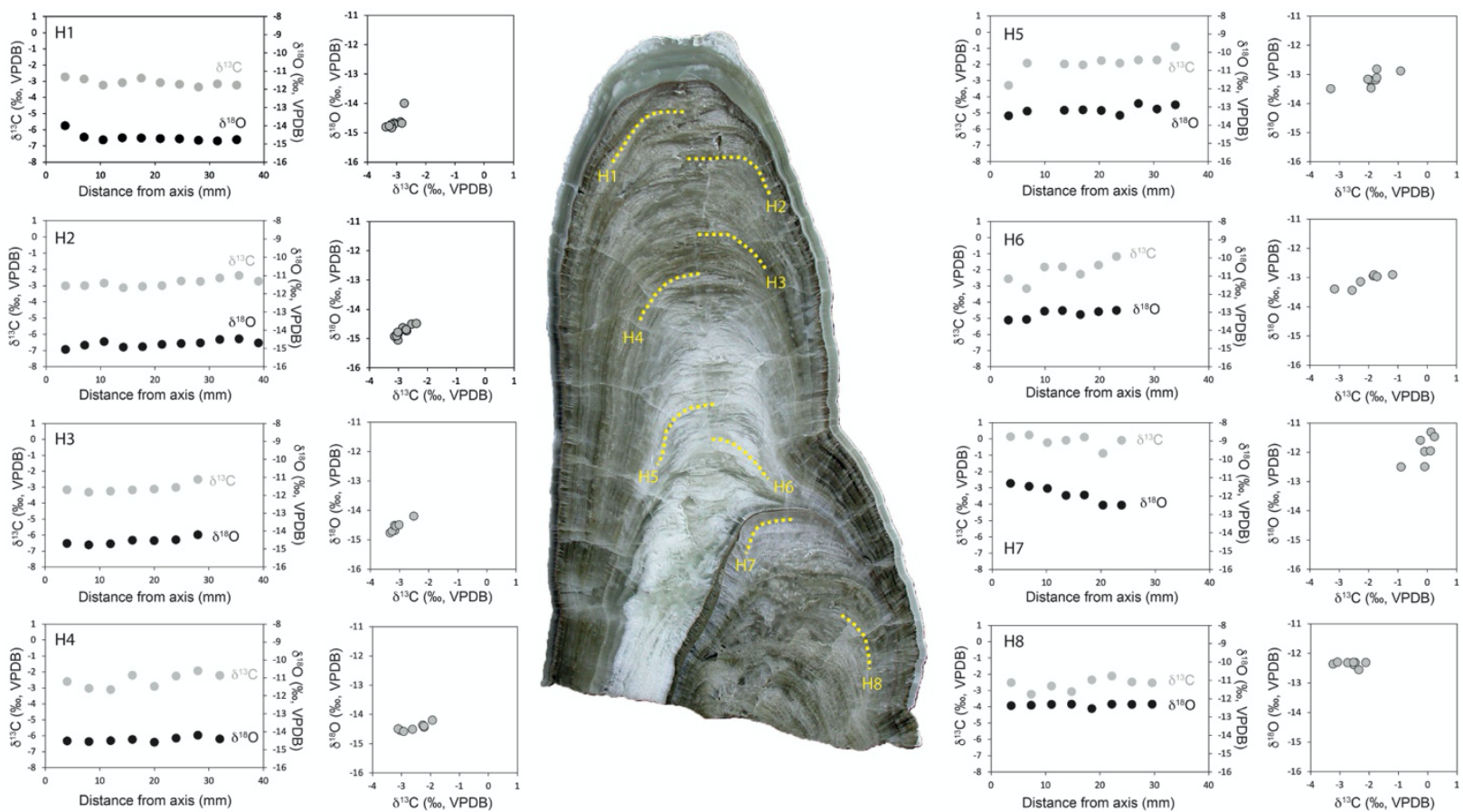

20

Figure S4: Hendy test results for stalagmite SPA146. Location of tests (H1-H8) are marked on the slab by yellow 21 dotted lines.

22

23 

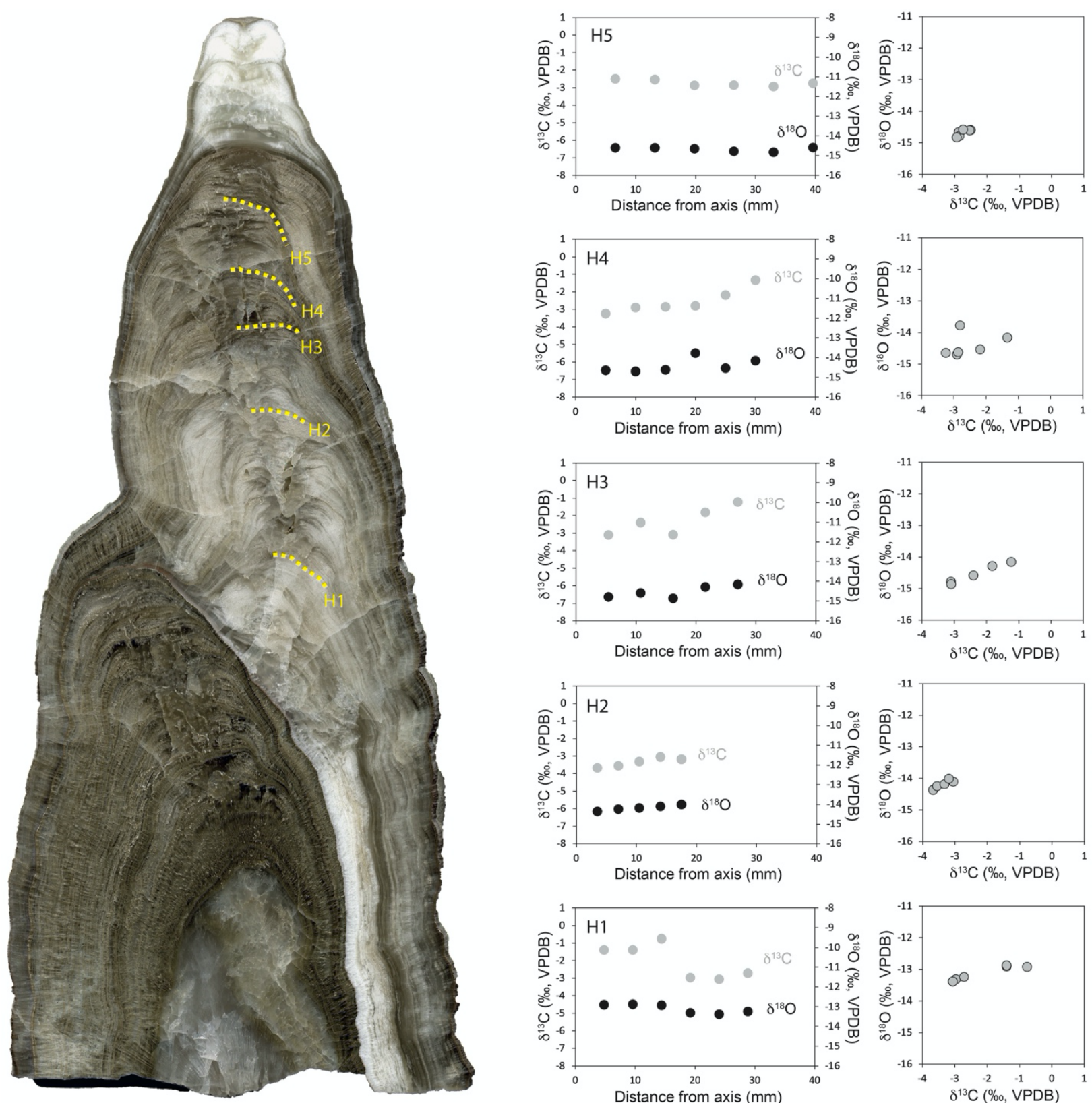

Figure S5: Supplementary figure 4: Hendy test results for stalagmite SPA183. Location of tests (H1-H5) are marked on the slab by yellow dotted lines.
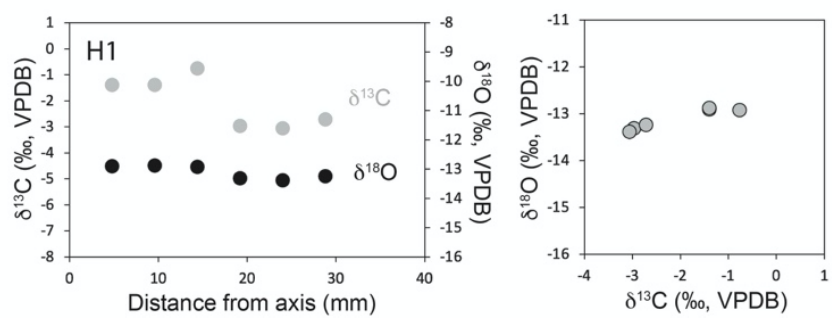
Supplementary materials for "Precise timing of MIS 7 substages from the Austrian Alps"

\begin{tabular}{|c|c|c|c|c|c|c|c|c|c|c|c|c|c|c|c|c|c|c|}
\hline \multirow{2}{*}{$\begin{array}{c}\text { Sample } \\
\text { ID } \\
\text { SPA121 }\end{array}$} & \multirow{2}{*}{$\begin{array}{l}\begin{array}{c}\text { DFT * } \\
\text { (mm) }\end{array} \\
48.45\end{array}$} & \multicolumn{2}{|c|}{$\begin{array}{l}{ }^{238} \mathrm{U} \\
(\mathrm{ppb})\end{array}$} & \multicolumn{2}{|c|}{$\begin{array}{l}{ }^{232} \mathrm{Th} \\
\text { (ppt) }\end{array}$} & \multicolumn{2}{|c|}{$\begin{array}{l}\delta^{234} \mathrm{U} * * \\
\text { (ס-units) }\end{array}$} & \multicolumn{2}{|c|}{$\begin{array}{l}{ }^{230} \mathrm{Th} /{ }^{232} \mathrm{Th} \\
\left(\text { atomic } \cdot 10^{-6} \text { ) }\right.\end{array}$} & \multicolumn{2}{|c|}{$\begin{array}{c}{ }^{230} \mathrm{Th} /{ }^{238} \mathrm{U} \\
\text { (activity) }\end{array}$} & \multicolumn{2}{|c|}{$\begin{array}{l}{ }^{230} \text { Th Age (yr) } \\
\text { (uncorrected) }\end{array}$} & \multicolumn{2}{|c|}{$\begin{array}{l}{ }^{230} \text { Th Age }(\mathrm{yr}) \\
\text { (corrected)*** }\end{array}$} & \multicolumn{2}{|c|}{$\begin{array}{c}\delta^{234} U_{\text {Initial }} * * * * \\
(\delta \text {-units })\end{array}$} & \multirow[t]{2}{*}{ Comments } \\
\hline & & 7968 & \pm 24 & 1768 & \pm 5 & -7.0 & \pm 0.4 & 51,278 & \pm 20 & 0.68983 & \pm 0.00034 & 129,658 & \pm 174 & 129,700 & \pm 174 & -10.1 & \pm 0.6 & \\
\hline SPA121 & 51.5 & 11826 & \pm 36 & 77.2 & 0.3 & -114.7 & \pm 0.2 & $1,794,848$ & \pm 4171 & 0.71056 & \pm 0.00034 & 191,338 & \pm 298 & 191,300 & \pm 298 & -196.8 & \pm 0.3 & \\
\hline SPA121 & 55.05 & 4611 & \pm 40 & 458 & \pm 4 & -111.5 & \pm 1.0 & 119,160 & \pm 96 & 0.71832 & \pm 0.00059 & 194,908 & \pm 963 & 194,900 & \pm 963 & -193.2 & \pm 1.8 & \\
\hline SPA121 & 60.45 & 5365 & \pm 22 & 1857 & \pm 8 & -112.6 & \pm 0.1 & 34,284 & \pm 9 & 0.71975 & \pm 0.00020 & 197,012 & \pm 192 & 197,000 & \pm 192 & -196.3 & \pm 0.2 & \\
\hline SPA121 & 67.95 & 544 & \pm 4 & 2605 & \pm 17 & -114.2 & \pm 0.1 & 2,488 & \pm 1 & 0.72294 & \pm 0.00020 & 201,144 & \pm 215 & 201,000 & \pm 248 & -201.4 & \pm 0.3 & \\
\hline SPA121 & 80.25 & 4704 & \pm 19 & 241 & \pm 1 & -117.8 & \pm 0.2 & 232,223 & \pm 280 & 0.72254 & \pm 0.00045 & 204,110 & \pm 439 & 204,100 & \pm 439 & -209.6 & \pm 0.4 & \\
\hline SPA121 & 81.5 & 4440 & \pm 9 & 90.4 & \pm 0.2 & -116.6 & \pm 0.7 & 587,734 & \pm 180 & 0.72577 & \pm 0.00028 & 205,909 & \pm 676 & 205,900 & \pm 676 & -208.5 & \pm 1.2 & \\
\hline SPA121 & 83.7 & 5817 & \pm 20 & 398 & \pm 1 & -114.0 & \pm 0.2 & 176,846 & \pm 178 & 0.73463 & \pm 0.00030 & 211,533 & \pm 340 & 211,500 & \pm 340 & -207.0 & \pm 0.4 & \\
\hline SPA121 & 91.95 & 6600 & \pm 18 & 919 & \pm 3 & -111.2 & \pm 0.2 & 87,484 & \pm 78 & 0.73921 & \pm 0.00045 & 213,138 & \pm 462 & 213,100 & \pm 462 & -203.0 & \pm 0.4 & \\
\hline SPA121 & 106.95 & 9049 & \pm 50 & 1042 & \pm 6 & -112.0 & \pm 0.2 & 106,046 & \pm 38 & 0.74064 & \pm 0.00027 & 215,316 & \pm 316 & 215,300 & \pm 316 & -205.6 & \pm 0.3 & \\
\hline SPA121 & 115.2 & 8471 & \pm 37 & 801 & \pm 3 & -107.9 & \pm 0.1 & 130,187 & \pm 335 & 0.74624 & \pm 0.00088 & 216,448 & \pm 879 & 216,400 & \pm 879 & -198.7 & \pm 0.5 & \\
\hline SPA121 & 119.7 & 5582 & \pm 20 & 721 & \pm 3 & -104.5 & \pm 0.2 & 95,699 & \pm 36 & 0.75015 & \pm 0.00030 & 216,779 & \pm 341 & 216,800 & \pm 341 & -192.7 & \pm 0.3 & \\
\hline SPA121 & 132.45 & 12151 & \pm 33 & 115.1 & 0.3 & -100.7 & \pm 0.2 & $1,317,303$ & \pm 1119 & 0.75713 & \pm 0.00022 & 219,543 & \pm 278 & 219,500 & \pm 278 & -187.0 & \pm 0.3 & \\
\hline SPA121 & 137 & 10734 & \pm 40 & 62.1 & \pm 0.2 & -108.9 & \pm 0.1 & $2,155,246$ & \pm 1062 & 0.75651 & \pm 0.00015 & 228,461 & \pm 229 & 228,500 & \pm 229 & -207.5 & \pm 0.3 & \\
\hline SPA121 & 142.2 & 12559 & \pm 37 & 434 & \pm 1 & -108.1 & \pm 0.2 & 362,477 & \pm 63 & 0.75994 & \pm 0.00016 & 231,348 & \pm 273 & 231,300 & \pm 273 & -207.7 & \pm 0.3 & \\
\hline SPA121 & 146.7 & 19031 & \pm 95 & 1909 & \pm 10 & -104.2 & \pm 0.2 & 126,242 & \pm 21 & 0.76807 & \pm 0.00018 & 235,745 & \pm 315 & 235,700 & \pm 315 & -202.7 & \pm 0.4 & \\
\hline SPA121 & 151.95 & 8291 & \pm 21 & 6023 & \pm 15 & -103.6 & \pm 0.1 & 17,484 & \pm 5 & 0.77024 & \pm 0.00016 & 237,565 & \pm 270 & 237,500 & \pm 271 & -202.6 & \pm 0.3 & \\
\hline SPA121 & 166.95 & 7963 & \pm 17 & 3632 & \pm 8 & -101.6 & \pm 0.1 & 28,003 & \pm 6 & 0.77450 & \pm 0.00014 & 240,013 & \pm 233 & 240,000 & \pm 233 & -200.1 & \pm 0.3 & \\
\hline SPA121 & 172.2 & 11373 & \pm 34 & 568 & \pm 2 & -100.6 & \pm 0.1 & 256,448 & \pm 111 & 0.77719 & \pm 0.00023 & 241,878 & \pm 349 & 241,900 & \pm 349 & -199.0 & \pm 0.3 & \\
\hline SPA121 & 176.7 & 13101 & \pm 38 & 381 & \pm 1 & -103.1 & \pm 0.1 & 438,800 & \pm 84 & 0.77461 & \pm 0.00012 & 242,182 & \pm 239 & 242,200 & \pm 239 & -204.1 & \pm 0.3 & \\
\hline SPA121 & 179 & 7146 & \pm 41 & 294 & \pm 2 & -104.7 & \pm 0.2 & 309,300 & \pm 100 & 0.77291 & \pm 0.00025 & 242,367 & \pm 449 & 242,400 & \pm 449 & -207.5 & \pm 0.5 & \\
\hline SPA121 & 184.2 & 14267 & \pm 100 & 1423 & \pm 10 & -101.4 & \pm 0.1 & 128,412 & \pm 39 & 0.77694 & \pm 0.00022 & 242,679 & \pm 331 & 242,700 & \pm 331 & -201.0 & \pm 0.3 & \\
\hline SPA121 & 187.20 & 13014 & \pm 45 & 1269 & \pm 4 & -101.6 & \pm 0.1 & 131,929 & \pm 27 & 0.78007 & \pm 0.00016 & 247,158 & \pm 287 & 247,200 & \pm 287 & -204.2 & \pm 0.3 & \\
\hline SPA121 & 190.95 & 12265 & \pm 50 & 2350 & \pm 10 & -102.8 & \pm 0.1 & 67,046 & \pm 16 & 0.77904 & \pm 0.00019 & 247,540 & \pm 329 & 247,500 & \pm 329 & -206.7 & \pm 0.3 & \\
\hline SPA121 & 193.8 & 11327 & \pm 41 & 10467 & \pm 38 & -101.1 & \pm 0.1 & 13,930 & \pm 10 & 0.78067 & \pm 0.00017 & 247,160 & \pm 274 & 247,100 & \pm 275 & -203.1 & \pm 0.3 & \\
\hline SPA146 & 15 & 71975 & \pm 6 & 118294 & \pm 11 & 20.4 & \pm 0.3 & 8,351 & \pm 14 & 0.83235 & \pm 0.00045 & 182,359 & \pm 301 & 182,300 & \pm 302 & 34.0 & \pm 0.5 & \\
\hline SPA146 & 73 & 17117 & \pm 126 & 71739 & \pm 530 & 188.3 & \pm 0.2 & 3,982 & \pm 5 & 1.01197 & \pm 0.00034 & 188,376 & \pm 193 & 188,300 & \pm 204 & 320.3 & \pm 0.5 & \\
\hline SPA146 & 101 & 17357 & \pm 154 & 23985 & \pm 218 & 180.3 & \pm 0.3 & 11,988 & \pm 25 & 1.00474 & \pm 0.00049 & 188,759 & \pm 264 & 188,700 & \pm 265 & 307.1 & \pm 0.5 & \\
\hline SPA146 & 115 & 17722 & \pm 495 & 11210 & \pm 313 & 200.8 & \pm 0.3 & 26,885 & \pm 8 & 1.03135 & \pm 0.00032 & 191,502 & \pm 224 & 191,500 & \pm 224 & 344.7 & \pm 0.6 & \\
\hline SPA146 & 154 & 57825 & \pm 9 & 3039 & \pm 1 & 165.9 & \pm 0.3 & 312,313 & \pm 304 & 0.99529 & \pm 0.00036 & 191,197 & \pm 236 & 191,200 & \pm 236 & 284.6 & \pm 0.6 & altered \\
\hline SPA146 & 162 & 66265 & \pm 12 & 8706 & \pm 2 & 161.8 & \pm 0.3 & 124,491 & \pm 136 & 0.99210 & \pm 0.00040 & 191,675 & $\pm 26 I$ & 191,700 & \pm 261 & 277.9 & \pm 0.6 & altered \\
\hline SPA146 & 180 & 42731 & \pm 3 & 2421 & \pm 2 & 185.4 & \pm 0.3 & 295,821 & \pm 787 & 1.01608 & \pm 0.00076 & 191,702 & \pm 400 & 191,700 & \pm 400 & 318.4 & \pm 0.7 & altered \\
\hline SPA183 & 85 & 20686 & \pm 353 & 138708 & \pm 2367 & 163.8 & \pm 0.3 & 2,440 & \pm 1 & 0.99199 & \pm 0.00053 & 190,649 & \pm 306 & 190,500 & \pm 324 & 280.4 & \pm 0.6 & \\
\hline SPA183 & 138.8 & 18743 & \pm 406 & 10928 & \pm 237 & 161.0 & \pm 0.2 & 27,988 & \pm 9 & 0.98970 & \pm 0.00032 & 190,908 & \pm 187 & 190,900 & \pm 187 & 275.9 & \pm 0.4 & \\
\hline SPA183 & 140 & 2135 & \pm 3 & 2908 & \pm 4 & 169.5 & \pm 0.2 & 12,125 & \pm 19 & 1.00172 & \pm 0.00037 & 192,578 & \pm 214 & 192,600 & \pm 215 & 291.8 & \pm 0.4 & \\
\hline SPA183 & 160 & 19652 & \pm 335 & 5839 & \pm 100 & 174.9 & \pm 0.2 & 55,716 & \pm 13 & 1.00405 & \pm 0.00025 & 191,040 & \pm 150 & 191,000 & \pm 151 & 299.8 & \pm 0.3 & \\
\hline SPA183 & 225.2 & 16905 & \pm 412 & 8469 & \pm 207 & 193.4 & \pm 0.2 & 33,686 & \pm 22 & 1.02449 & \pm 0.00062 & 191,806 & \pm 314 & 191,800 & \pm 314 & 332.3 & \pm 0.5 & \\
\hline SPA183 & 220 & 26714 & \pm 2 & 12903 & \pm 2 & 252.1 & \pm 0.3 & 36,934 & \pm 73 & 1.08175 & \pm 0.00069 & 190,760 & \pm 336 & 190,800 & \pm 336 & 431.8 & \pm 0.7 & altered \\
\hline SPA183 & 240 & 100414 & \pm 14 & 47225 & \pm 7 & 134.6 & \pm 0.3 & 33,811 & \pm 34 & 0.96444 & \pm 0.00031 & 191,673 & \pm 228 & 191,700 & \pm 228 & 231.2 & \pm 0.6 & altered \\
\hline
\end{tabular}




\section{Supplementary materials for "Precise timing of MIS 7 substages from the Austrian Alps"}

Table S1: U-Th dating results for Spannagel stalagmites included in this study. Uncertainties are $2 \sigma$. U decay constants: $\lambda_{238}=1.55125 \times 10^{-10}$ (Jaffey et al., 1971) and $\lambda_{234}=2.82206 \times 10^{-6}$ (Cheng et al., 2013). Th decay constant: $\lambda_{230}=9.1705 \times 10^{-6}$ (Cheng et al., 2013). Corrected ${ }^{230} \mathrm{Th}$ ages assume the initial ${ }^{230} \mathrm{Th} /{ }^{232} \mathrm{Th}$ atomic ratio of $4.4 \pm 2.2$ $\mathrm{x} 10^{-6}$. Those are the values for a material at secular equilibrium, with the bulk earth ${ }^{232} \mathrm{Th} /{ }^{238} \mathrm{U}$ value of 3.8. The errors are arbitrarily assumed to be $50 \%$. DFT* $=$ distance from top. ${ }^{* *} \delta^{234} \mathrm{U}=\left(\left[{ }^{234} \mathrm{U} /{ }^{238} \mathrm{U}\right]\right.$ activity -1$) \mathrm{x} 1000 .{ }^{* * *} \mathrm{BP}$ stands for "Before Present" where the "Present" is defined as the year 1950 A.D. **** $\delta^{234} U_{\text {initial }}$ was calculated based on ${ }^{230} \mathrm{Th}$ age $(\mathrm{T})$, i.e. $\delta^{234} \mathrm{U}_{\text {initial }}=\delta^{234} \mathrm{U}_{\text {measured }} \mathrm{X} \mathrm{e}^{(\lambda 234)(\mathrm{T})}$. Italicized ages were sampled within the altered portions of stalagmites SPA146 and 183 (see text for details), and were not included in final age models.

\section{Supplementary material references}

Cheng, H., Edwards, R. L., Shen, C. C., Polyak, V.J., Asmerom, Y., Woodhead, J., Hellstrom, J., Wang, Y., Kong, X., Spötl, C., Wang, X., and Alexander, E. C.: Improvements in ${ }^{230}$ Th dating, ${ }^{230} \mathrm{Th}$ and ${ }^{234} \mathrm{U}$ halflife values, and U-Th isotopic measurements by multi-collector inductively coupled plasma mass spectrometry, Earth Planet. Sci. Lett., 371, 82-91, doi:10.1016/j.epsl.2013.04.006, 2013.

Jaffey, A. H., Flynn, K. F., Glendenin, L. E., Bentley, W. C., Essling, A. M.: Precision measurement of halflives and specific activities of $235 \mathrm{U}$ and 238, Physical Review, 5, 1889-1906, https://doi.org/10.1103/PhysRevC.4.1889, 1971. 